\title{
Case report: Unusual complications of severe hidradenitis suppurativa
}

\author{
S R Gautam, MB ChB; A E Zühlke, MD \\ Division of Plastic and Reconstructive Surgery, Faculty of Medicine and Health Sciences, Stellenbosch University and Tygerberg Academic Hospital, Cape Town, \\ South Africa
}

Corresponding author: SR Gautam (srgautam84@gmail.com)

\begin{abstract}
Hidradenitis suppurativa/acne inversa (HS/Al) is a chronic follicular occlusive disease that affects the folliculopilosebaceous unit, mainly, but not exclusively, in intertriginous axillary, groin, perianal, perineal, genital and inframammary skin. The clinical course is highly variable. The disease is difficult to treat, and has a severe impact on quality of life. An 18-year-old female was diagnosed with bilateral axillary HS/Al in 2014. She was a known smoker, with a body mass index of 21, no known comorbidities and retroviral-disease negative. She presented in 2017 with advanced Hurley stage III disease, with extensive painful open wounds and purulent material draining. The patient also had bilateral shoulder contractures with reduced abduction. She was clinically malnourished, anaemic and hypoalbuminaemic. Wound swabs showed a Pseudomonas infection. Wide local excision was performed of the diseased areas and skin grafted, and arm splints were designed to keep both arms in fixed abduction, to allow for adequate healing and wound care management. However, all grafts failed to take. We employed a multidisciplinary approach to manage this patient. Negative-pressure wound therapy was utilised to assist with wound management. The patient had developed a spontaneous pneumothorax owing to the severity of the disease, which eroded into the chest wall. This was managed conservatively with an intercostal drain. Despite all these efforts, the patient's condition did not improve. She subsequently went into septic shock, and died in the intensive care unit. This case illustrates the fact that early diagnosis and management of HS/Al is essential. As the disease advances, it becomes increasingly difficult to manage, and requires a multidisciplinary treatment approach.
\end{abstract}

S Afr J Plast Reconstr Aesthet Surg Burns 2018;1(1):21-22. DOI: 10.7196/SAJPRASB.2018.1.1.1

Hidradenitis suppurativa (HS, from the Greek hidros = sweat, and aden $=$ glands) is a chronic inflammatory skin condition that is also known as acne inversa (Al). ${ }^{[1]} \mathrm{HS} / \mathrm{Al}$ is a chronic follicular occlusive disease involving the follicular portion of folliculopilosebaceous units (FPSUs). ${ }^{[1]}$ The prevalence of HS/AI has varied, ranging from less than $1 \%$ to $4 \%{ }^{[2]}$ The onset of symptoms typically occurs between puberty and age 40 , most commonly in the second or third decade of life. ${ }^{[2]}$ The incidence of $\mathrm{HS} / \mathrm{Al}$ is twice as high among women than men. ${ }^{[2]}$ Genetic susceptibility, mechanical stresses on the skin, obesity, smoking, diet and hormonal imbalances are repeatedly cited as factors that may be associated with the development or exacerbation of HS/Al. ${ }^{[3]}$ The primary sites of involvement for $\mathrm{HS} / \mathrm{Al}$ are the intertriginous skin areas of the axillary (61\%), groin (48\%), perianal $(30-50 \%)$, perineal $(22 \%)$ and inframammary regions (13\%), though it can occur in any skin area that contains FPSUs. ${ }^{[2]}$ The clinical manifestations vary, ranging from recurrent inflamed nodules and abscesses to draining sinus tracts and bands of severe scar formation. ${ }^{[3]}$ The associated pain, malodour, drainage and disfigurement that accompany HS/AI contribute to its profound psychosocial impact on many patients. ${ }^{[3]}$

\section{Case report}

An 18-year-old female was diagnosed with bilateral axillary HS in 2014. She was a known smoker, with a body mass index of 21, and no known medical comorbidities.

\section{Clinical findings}

She presented in 2017 with advanced Hurley stage III disease, with extensive painful open wounds extending from both axillae onto the chest wall and upper arms, and threatening to erode the left brachial vessels (Fig.1). The patient also had severe bilateral shoulder adduction contractures and reduced function, with oedema of the left arm. The perineum, perianal and groin regions were not involved.

\section{Laboratory investigations}

Laboratory tests showed: white cell count of $27.25 \times 109 / \mathrm{L}$ with predominant neutrophils; haemoglobin $7.8 \mathrm{~g} / \mathrm{dL}$; haematocrit 15\%; platelet

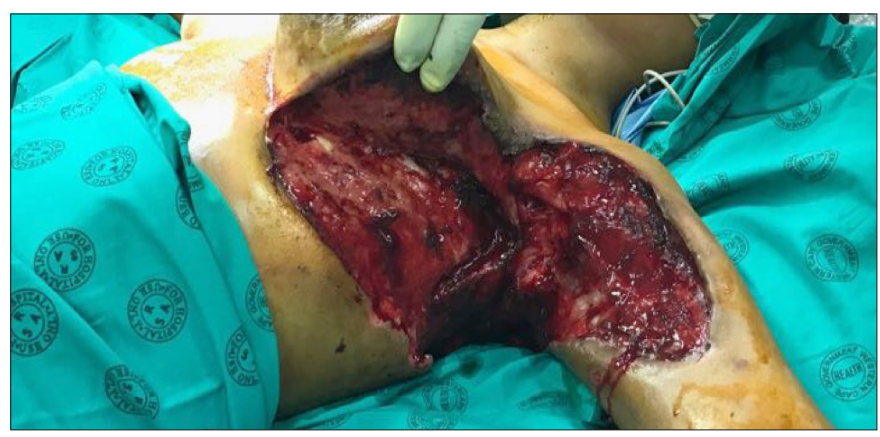

Fig. 1. Severe hidradenitis suppurativa (Hurley stage III) of the left axilla, chest wall and upper arm. 
count $254 \times 109 / \mathrm{L} ; \mathrm{C}$-reactive protein $132 \mathrm{mg} / \mathrm{L}$; serum renal function and electrolytes normal; liver function tests normal; and hypoalbuminaemia with an albumin of $12 \mathrm{~g} / \mathrm{L}$. All immune studies (rheumatoid factor, complement C3, complement C4, ANF and AntidsDNA Ab) were normal. She was retroviral disease-negative. Wound swabs of both axillae showed a Pseudomonas aeruginosa sensitive to Piptaz and carbapenem.

\section{Management}

During the course of her treatment, we employed a multidisciplinary team approach to manage this patient (psychologist, psychiatrist, dermatologist, dietitian, occupational therapist, physiotherapist, internal medicine physician, social worker, orthopaedic surgeon, cardiothoracic surgeon, abdominal surgeon and intensivist). After wide local debridement, supportive treatment (dietitian, nasogastric tube, percutaneous endoscopic gastrostomy feeding tube, occupational therapist, physiotherapist, psychiatrist) and wound conditioning (antibiotic treatment, negative-pressure wound therapy), the defects were skin grafted. Arm splints were designed to keep both arms in fixed abduction, to allow for adequate healing and wound care management. However, the wounds showed no signs of healing, grafts failed to take and under a negative pressure wound therapy (NPWT) dressing, the disease eroded into the chest wall, causing a pneumothorax. This was demonstrated on chest X-ray. Ultrasound was negative for any intra-abdominal findings. Despite all supporting and surgical efforts, the patient's condition deteriorated. She developed overwhelming sepsis, and was transferred to the intensive care unit (ICU). Despite extensive resuscitative efforts, the patient died in the ICU.

\section{Discussion}

$\mathrm{HS} / \mathrm{Al}$ is difficult to treat owing to its complex pathomechanism; beside the extensive inflammation with abscesses and inflammatory nodules, there are also sinus-tract formations and, in severe cases, extensive scarring. ${ }^{[3]}$ Axillary hidradenitis is often mild, but perineal HS/AI is often associated with frequent recurrences and poor outcomes. ${ }^{[4]} \mathrm{HS} / \mathrm{Al}$ does not usually involve the deeper tissue, because it travels along and is limited by the fascia in the involved sites. ${ }^{[4]}$ The clinical course is highly variable. The disease can perforate, although rarely, the rectum, urinary bladder, urethra and peritoneum. ${ }^{[4]}$ On review of the literature, one finds that fistula formation involving underlying organs is rare, and death as a result of this disease is very rare. ${ }^{[4]}$ Although it is a treatable disease, $\mathrm{HS} / \mathrm{Al}$ can lead to systemic sequelae severe enough to contribute directly to death. ${ }^{[4]} A$ PubMed search from 1966 to 2016 revealed only two deaths reported to be caused directly from benign HS/AI. ${ }^{[4]}$ In both cases, the patients had extensive perineal and perianal HS/Al with fistulous communication to pelvic organs..$^{[5]}$ Ultimately, the patients died of severe peritonitis, multiorgan failure and overwhelming sepsis after surgery. ${ }^{[5]}$ A fatality caused by axillary HS/Al has not been previously described. ${ }^{[5]}$ Other reported complications from HS/AI include anaemia, interstitial keratitis and osteomyelitis. ${ }^{[6]}$ Squamous cell carcinoma arising in HS/Al (perineal) is rare. ${ }^{[5]}$ Surgery should be introduced early in the management of HS/Al. Unfortunately, surgical treatment is often performed many years after the initial symptoms of the disease appear, after numerous ineffective cycles of pharmacotherapy. ${ }^{[5]}$ Such delays are caused by misdiagnosis, a doctor's lack of knowledge about the disease, unjustified confidence in the efficacy of non-invasive therapies, anxiety about surgical treatment and the embarrassing location. ${ }^{[5]}$ An early and accurate diagnosis facilitates the initiation of a treatment plan aimed at minimising the risk of progression to disabling, end-stage disease. ${ }^{[5]}$ The treatment of choice for advanced disease is early radical excision, with skin grafts or flaps, or healing by secondary intention. ${ }^{[4]}$ The end-stage disease is difficult to treat, and has a negative impact on quality of life. ${ }^{[5]}$ The combination of the extensive wounds paired with a catabolic state, and an unusual complication, led to a fatal result in this young patient. Pyoderma gangrenosum is one of the differential diagnoses in this specific case. It is an uncommon neutrophilic dermatosis that most commonly presents with inflammatory ulcers on the skin. ${ }^{[6]}$ This disease entity may occur at any age. ${ }^{[6]}$ More than $50 \%$ of patients with pyoderma gangrenosum have an associated systemic disease. ${ }^{[6]}$ Since the clinical and histological findings of pyoderma gangrenosum are non-specific, it is a diagnosis of exclusion. ${ }^{[6]}$ Pyoderma gangrenosum is extremely difficult to treat, and it often needs the association of systemic treatments combined with a correct wound-bed preparation approach ${ }^{[6]}$ Therefore, on clinical presentation and histological findings, pyoderma gangrenosum was not confirmed in this case.

\section{Acknowledgements. None. \\ Author contributions. Equal contributions. \\ Funding. None. \\ Conflicts of interest. None.}

Saunte DML, Jemec GBE. Hidradenitis suppurativa: Advances in diagnosis and treatment. JAMA 2017;318(20):20192032. https://doi.org/10.1001/jama.2017.1669.

2. Ingram JR. Hidradenitis suppurativa (acne inversa): Pathogenesis, clinical features, and diagnosis. Post TW, ed. UpToDate. Waltham, MA: UpToDate Inc. https://www.uptodate.com/contents/hidradenitis-suppurativapathogenesis-clinical-features-and-diagnosis (accessed 1 September 2018).

3. Jemec GBE. Hidradenitis suppurativa. N Engl J Med 2012;366(2):158-164. https://doi.org/10.1056/ NEJMcp 1014163

4. Groginsky EM, Olson JK, Lallas TA, Buekers TE, Potts S, Sorosky Jl. A case of severe hidradenitis suppurativa contributing to a death, and a review of the literature. J Low Genit Tract Dis 1999;3(1):67-76.

5. Moschell SL. Hidradenitis suppurativa. Complications resulting in death. JAMA 1966;198(1):201-203.

6. Schadt C. Pyoderma gangrenosum: Pathogenesis, clinical features and diagnosis. Post TW, ed UpToDate Waltham, MA. UpToDate Inc htps//wmpw uptodatecom/contents/pyoderma gangrenosumpathogenesisclinical-features-and-diagnosis (accessed 7 November 2018).

Accepted 8 November 2018. 